Starkstein, S.E., Brockman, S. \& Hayhow, B.D. (2012). Psychiatric syndromes in Parkinson's disease. CURRENT OPINION IN PSYCHIATRY, 25(6), 468-472.

Copyright: @ 2012 Lippincott Williams \& Wilkins, Inc.

This is pre-copy-editing, author-produced version of an article accepted for publication in Current Opinion In Psychiatry following peer review. The definitive published version (see citation above) is located on the home page of the publisher, Lippincott Williams and Wilkins. (http://www.co-psychiatry.com)

This version will be made available in the UWA Research Repository on the $1^{\text {st }}$ of December 2013, in compliance with the publisher's policies on archiving in institutional repositories.

Use of the article is subject to copyright law. 


\title{
Psychiatric syndromes in Parkinson's disease
}

\author{
Sergio E. Starkstein, Simone Brockman, Bradleigh D. Hayhow \\ School of Psychiatry and Neurosciences, University of Western Australia \\ Western Australia, Australia
}

Acknowledgments: This article has been partially supported from grants from NHMRC (Australia), and the Michael J. Fox Foundation (USA).

Conflicts of interests: There are no conflicts of interest

Send correspondence to:

Professor Sergio Starkstein

School of Psychiatry

University of Western Australia

Fremantle Hospital T-7

Phone: 61-8-9431-2013

E-mail: Sergio.starkstein@uwa.edu.au 


\begin{abstract}
Purpose of review

To examine progress about relevant behavioural and psychiatric disorders in Parkinson's disease (PD), such as depression, apathy, psychosis, and impulse control disorder.
\end{abstract}

\title{
Recent findings
}

Several recent studies have characterized the phenomenology of depression in PD, and randomized controlled trials have demonstrated the efficacy of tricyclics, selective serotonin reuptake inhibitors (SSRIs) and psychotherapy for depression in PD. Apathy is a valid behavioural syndrome in PD and is associated with depression and cognitive deficits. Psychosis is highly prevalent in the late stages of the disease, but there are few effective therapeutic modalities for this psychiatric condition. Impulse control disorders are also relatively frequent in PD, and are associated with co-morbid psychiatric disorders.

\section{Summary}

Standardized criteria should be used to diagnose depression and apathy in PD. Psychotherapy and pharmacotherapy are useful treatment modalities for affective disorders in PD. Clozapine is still the most effective, albeit rarely used, treatment for psychosis in PD. Impulsive control disorders are relatively frequent in PD and all patients should be screened for this complex disorder.

\section{Key-words}

Parkinson's disease, depression, apathy, psychosis, impulse control disorders 


\section{INTRODUCTION}

The past 20 years have witnessed a geometric increase in studies about the phenomenology, epidemiology, clinical correlates, mechanism and treatment of psychiatric syndromes in Parkinson's disease (PD). This review will provide recent information on advances in depression, apathy, psychosis and impulsive behaviours.

\section{RECENT ADVANCES IN DEPRESSION}

Depression is found in about 20 to $40 \%$ of cross-sectional samples with PD (1), and is associated with a faster cognitive and functional decline, and a faster progression along the stages of the illness (2).

\section{Phenomenology}

The diagnosis of depression in PD is complicated by the overlap between physical symptoms of PD and psychological symptoms of depression. To clarify this overlap and provide clear diagnostic guidelines, Starkstein and co-workers (3)* examined the validity of specific criteria for depression in a series of 259 patients with PD. Using latent class analysis and structured psychiatric interviews, the authors found that all 8 DSM-IV criteria for major depression identified a class of patients with a high frequency of depressive symptoms, suggesting that DSM-IV criteria for major depression may be used unmodified in PD. There was a second class with a low frequency of all depressive symptoms, demonstrating that the presence of motor symptoms in PD does not "inflate" the rate of symptoms of depression. Finally, there was a third class with an intermediate frequency of depressive symptoms, which included patients meeting DSM-IV criteria for major depression, minor depression, or dysthymia. Patients in the classes with severe or moderate depression had a significantly higher frequency of apathy as compared to the class with no depression, and there was a graded increase in anxiety symptoms (more common in the class with severe depression). 
Brown and co-workers (4) examined the presence of subtypes of PD based on the presence of anxiety and depression using latent class analysis. The study identified four classes. The largest class (60\% of participants) was characterized by low levels of both anxiety and depression. A second class included $22 \%$ of the sample and showed prominent anxiety, whilst the remaining two classes showed high possibilities of core symptoms of depression, one with associated anxiety symptoms and the other with associated apathy symptoms. Longitudinal studies are needed to examine the stability of the latent classes identified by Starkstein's and Brown's studies.

The potential overlap between parkinsonism and psychological symptoms of depression was recently examined in a study that recruited participants to a randomised controlled trial (RCT) of nortriptyline and paroxetine (5). The authors found that treatment responders showed significant improvements on depressed mood, middle insomnia, loss of interest and somatic anxiety. Furthermore, responders showed a 50\% improvement on 15 of the 16 HAM-D items, suggesting that all symptoms of depression may be used for diagnosis in PD.

\section{Treatment of depression}

Several RCTs using pharmacotherapy or psychotherapy for depression in PD have been recently reported. Dobkin and co-workers (6) carried out the first RCT of cognitive behavioural therapy (CBT) in PD with depression. Participants in the active arm received 10 weekly individual sessions of manualized $\mathrm{CBT}$, which consisted of exercises, behavioural activation, thought monitoring and restructuring, relaxation training, worry control, sleep hygiene and specific education sessions for caregivers. The placebo arm consisted of clinical monitoring only. The main finding was that patients receiving CBT showed a significantly greater reduction on depression scores than the placebo group, with a treatment response of $56 \%$ for CBT and $8 \%$ for placebo. Moreover, CBT was also related with improvements on secondary outcome measures such as anxiety, social functioning, and motor problems. One limitation is that the mean Hamilton Depression Scale (HAM-D) score at the final 
assessment for the CBT group was 13 points, suggesting that a subgroup of patients still had moderate depression. Future studies should also examine the efficacy of CBT among PD patients with early dementia or severe depression, which were excluded from the trial.

Weintraub and co-workers (7) examined the efficacy of the selective norepinephrine reuptake inhibitor atomoxetine in a RCT that included 55 depressed PD patients. The group receiving atomoxetine showed significant improvements on secondary outcome measures such as global cognition and daytime sleepiness, but not on depression. The efficacy of the dopamine agonist pramipexole $(0.125 \mathrm{mg}$ to $1 \mathrm{mg})$ to treat depression in PD (8) was examined in a multi-centre 12-week RCT. There was a significant decline in depression scores in the pramipexole group which remained significant after adjusting for motor improvement. Nevertheless, the adjusted mean difference on depression scores between the active and placebo groups was of only 2 points, which is of unclear clinical significance. The rate of responders was $38 \%$ in the pramipexole group and $23 \%$ in the placebo group $(p<0.01)$, suggesting that most PD patients with mild or moderate depression do not respond to pramipexole. Tolerability was good, and more frequent dyskinesias in the pramipexole group were the only difference in terms of side-effects as compared with placebo.

SSRIs are the most commonly used antidepressants in PD, but recent studies suggest that tricyclic antidepressants have the same or even higher efficacy. Devos and co-workers (9) compared the efficacy of desipramine (up to $75 \mathrm{mg} / \mathrm{d}$ ) and citalopram (up to $20 \mathrm{mg} / \mathrm{d}$ ) in a 4-week RCT that included 40 patients with PD and major depression. Both antidepressants showed significant efficacy over placebo for depression, but all three groups showed significant improvements on anxiety scores. Relevant adverse events were reported by 2 patients on citalopram and 1 patient on desipramine, but acceptability for citalopram was twice as high as that for desipramine. Menza and co-workers (10) carried out an eight-week RCT that compared nortriptyline (up to $75 \mathrm{mg} / \mathrm{d}$ ), paroxetine (up to $37.5 \mathrm{mg} / \mathrm{d}$ ) and placebo. The study included 52 PD patients with major depression or dysthymia. The main finding was that nortriptyline was significantly more effective than both paroxetine and placebo, whilst paroxetine was no more effective than placebo. The rate of 
responders was $53 \%$ for nortriptyline, $11 \%$ for paroxetine and $24 \%$ for placebo. Patients on nortriptyline also showed significant improvements on secondary outcome measures, such as quality of life, sleep, and anxiety, but there were no significant between-group differences on UPDRS-motor and cognition scores. Side effects on nortiptyline were mild to moderate, and acceptability was comparable among the three groups. Another interesting finding from this study (11) was that responders showed higher baseline scores on measures of executive functions, speed of processing, and verbal memory. On the other hand, treatment responders did not show improved cognition at the end of the trial as compared to non-responders. Given that this study excluded patients with dementia, a ceiling effect cannot be ruled out. Another study provided further support for the use of tricyclics in PD. Paumier and co-workers (12)* found that patients with early PD treated with amitriptyline showed a significant delay in starting dopaminergic treatment as compared to patients with early PD on no tricyclics. A recent 12-week RCT compared the efficacy of paroxetine (up to 40 $\mathrm{mg} / \mathrm{d}$ ) and venlafaxine (up to $225 \mathrm{mg} / \mathrm{d}$ ) in 115 PD patients with either major depression, minor depression or dysthymia (13)**. At the end of the study, both paroxetine and venlafaxine showed greater efficacy than placebo. However, the remission rate was not significantly different (44\% for paroxetine, $37 \%$ for venlafaxine and $32 \%$ for placebo, whilst response rates were $68 \%, 53 \%$ and $44 \%$, respectively). Venlafaxine was associated with a significant increase in mean blood pressure, whilst paroxetine was associated with significant weight gain. Future studies will have to clarify the usefulness of paroxetine to treat depression in PD. The Movement Disorders Society (MDS) taskforce on evidence-based medicine (14) suggested pramipexole to be efficacious (i.e. evidence shows that intervention has a positive effect on studies outcomes). Nortriptyline and desipramine were considered likely efficacious (i.e. evidence suggests, but is not sufficient to demonstrate a positive effect on studies outcome). There were no major concerns with regards to safety, provided tricyclics were used at moderate doses. On the other hand, there was insufficient evidence for amitriptyline, citalopram, sertraline, paroxetine, fluoxetine, atomoxetine and rTMS. 
There is a single RCT that examined the efficacy of repeated transcranial magnetic stimulation (rTMS) for depression in PD. Pal and co-workers (15) applied a 10-day course of left prefrontal sub threshold rTMS or sham treatment in a sample of 22 patients with mild or moderate depression. Patients receiving active treatment showed a significant decrease in depression scores over time, whilst no changes on depression scores were observed in the sham group. Unfortunately, the authors did not compare depression score changes between the active and sham groups. The treatment response was $75 \%$ for the active group and $20 \%$ for the sham group, but the criterion for "response" was not specified.

\section{RECENT ADVANCES ON APATHY}

Apathy is a common finding in PD as is usually associated with increased depression and cognitive deficits. Its mechanism remains poorly understood, and no effective treatments have been identified.

\section{Diagnosis and correlates of apathy}

Apathy has been increasingly recognized as a common behavioural problem in PD. There are a variety of apathy rating scales that have been developed during the past 10 years, but standardised diagnostic criteria and valid diagnostic interviews are still lacking. Starkstein and co-workers (16) assessed the validity and nosological position of apathy in a study that included 164 patients with PD. Apathy was assessed with the Apathy Scale, and diagnoses of apathy were generated using a validated algorithm and diagnostic criteria. The study showed a strong agreement between diagnostic criteria and expert clinical diagnosis. Moreover, apathy in PD identified a group of patients with relatively more severe depression and greater functional deficits, but neither dementia nor depression is sufficient to produce apathy in PD. Dujardin and co-workers (17) reported that among PD patients without depression or dementia, apathy was a significant predictor of dementia. Similarly, Reijnders and co-workers (18) reported a significant association between apathy and both 
decreased cognitive functioning and more severe depression $(18,19)$. Using structural MRI they found that higher apathy scores were significantly correlated with low grey matter density in bilateral precentral, inferior parietal, and inferior frontal gyry, insula, and the right posterior cingulate and right precuneus.

\section{RECENT ADVANCES IN PSYCHOSIS}

Psychosis in PD has an overall prevalence of about 20 to $40 \%$ (19) and is associated with older age, use of antiparkinsonian medication, presence of dementia, more severe parkinsonism and comorbid depression. A recent longitudinal study that included patients in the early stages of PD (20) showed a 1-year prevalence of psychosis of $3 \%$, increasing to $7.7 \%$ two years later. The main predictors of psychosis were longer disease duration and use of dopamine agonists, but the study was limited by not controlling for the intake of antiparkinsonian medication during the follow-up period. Forsaa and co-workers (21) conducted a 12-year prospective longitudinal cohort study of 230 community based individuals with PD. By the end of the study $60 \%$ of the patients had developed psychotic symptoms, and the baseline independent predictors of psychosis were a higher age at onset of PD, a higher use of antiparkinsonian medication, and the presence of REM sleep behavioural disorder.

\section{Diagnosis of psychosis in PD}

The MDS task force on PD Rating Scales labelled the following instruments as recommended to assess psychotic symptoms in PD: the Neuropsychiatric Inventory, the Brief Psychotic Rating Scale, the Positive and Negative Syndrome Scale, and the Schedules for Assessment of Positive Symptoms (22). The Parkinson's Psychosis Rating Scale, the Parkinson's Psychosis Questionnaire and the Clinical Global Impression Scale were labelled as suggested. The recommended scales should be accompanied by a global measure of severity focused on psychosis. Nevertheless, the task force concluded that a new scale should be specifically developed to assess psychotic symptoms in PD. 


\section{Treatment of psychosis in PD}

Weintraub and co-workers (23)** examined the frequency and type of antipsychotic medication generally used in PD. This retrospective study included estimates from about 60,000 individuals with PD from the Veterans Administration system. About half of the patients with psychosis were prescribed antipsychotic medication, and the drug most commonly used was quetiapine, followed in frequency by risperidone, aripiprazole, olanzapine, ziprazidone, and clozapine. Surprisingly, $7 \%$ of patients received treatment with typical neuroleptics, and only $2 \%$ were on clozapine; the only atypical neuroleptic with established efficacy in PD. There was a decrease in use of olanzapine and risperidone over time, with increases in quetiapine and ziprazidone use. These findings suggest a shift to use better tolerated antipsychotics, albeit with questionable efficacy. The MDS task-force on treatment for non-motor symptom in PD (14) suggested that clozapine should be considered efficacious and its risk acceptable provided there is specialized monitoring. On the other hand, there was insufficient evidence for the use of quetiapine, whilst olanzapine was considered unlikely efficacious.

\section{RECENT ADVANCES IN IMPULSE CONTROL DISORDERS (ICD)}

Impulse Control Disorders (ICD) include pathological gambling, compulsive shopping, compulsive sexual behaviour, and binge eating, and these behavioural problems have been reported in about $14 \%$ of patients with PD (24).

\section{Epidemiology and comorbidity of ICD}

Few studies have examined the longitudinal evolution of ICD in PD. Avila and co-workers (25) followed a series of 25 PD patients with ICD for a median of 12 months. They found that 15 of 25 patients (83\%) who decreased their dopaminergic treatment had a partial or full remission of ICD. Weintraub and co-workers (26) found a significant association between ICD and use of amantadine, whilst Voon and co-workers $(27)^{* *}$ found ICD to be associated with greater functional impairment, 
more severe depression and anxiety, greater obsessive-compulsive symptoms, and greater impulsivity, suggesting that psychiatric comorbidity may contribute to the development of ICD in PD.

\section{Mechanism of ICD in PD}

Cilia and co-workers (28) examined the neural network that may characterize pathological gambling in a study that included 15 PD patients with pathological gambling and 15 patients without pathological gambling who underwent a resting single photon emission tomography scan. Patients with pathological gambling showed reduced perfusion in the right ventrolateral prefrontal cortex, anterior cingulate and striatum. The authors speculated that disconnection of the striatum from the anterior cingulate input may dispose to pathological gambling in PD.

\section{CONCLUSION}

Depression in PD can be diagnosed with high validity and specificity, and recent studies have demonstrated the efficacy of both psychotherapy and pharmacotherapy in treating this condition. Future studies should examine the efficacy of combining these treatment modalities. Apathy is well characterized and its nosological position validated. However, effective interventions have yet to be developed. Psychosis is a frequent problem among PD patients in the late stages of the disease. Unfortunately treatment recommendations do not match usual treatment in clinical practice and this important discrepancy should be addressed. Finally, ICD is increasingly recognized in PD. Its pathogenesis seems to be multifactorial, which may complicate treatment approaches. 


\section{KEY POINTS}

- Depression in PD should be diagnosed using unmodified DSM-IV criteria

- Depression in PD is effectively treated with Cognitive Behavioural Therapy and

pharmacotherapy (e.g. nortriptyline, venlafaxine).

- Apathy is common in PD and is associated with depression and cognitive deficits.

- Clozapine has the highest efficacy among antipsychotics to treat psychosis in PD but it is rarely used.

- Impulse Control Disorders are related to co-morbid psychiatric disorders and is more frequent among PD patients on amantadine. 


\section{REFERENCES AND RECOMMENDED READING}

1. Starkstein S, Merello M, Jorge R, Brockman S, Bruce D, Petracca G, et al. A validation study of depressive syndromes in Parkinson's disease. Movement Disorders. 2008;15:538-46.

2. Starkstein SE, Mayberg HS, Leiguarda R, Preziosi TJ, Robinson RG. A prospective longitudinal study of depression, cognitive decline, and physical impairments in patients with Parkinson's disease. Journal of Neurology, Neurosurgery \& Psychiatry. 1992;55(5):377-82.

3. Starkstein S, Dragovic M, Jorge R, Brockman S, Merello M, Robinson RG, et al. Diagnostic criteria for depression in Parkinson's disease: a study of symptom patterns using latent class analysis. Movement disorders : official journal of the Movement Disorder Society. 2011;26(12):223945.

* This study provided validation to specific criteria for diagnosing depression in Parkinson's disease.

4. Brown RG, Landau S, Hindle JV, Playfer J, Samuel M, Wilson KC, et al. Depression and anxiety related subtypes in Parkinson's disease. Journal of Neurology Neurosurgery and Psychiatry. 2011:in press.

5. Dobkin RD, Menza M, Bienfait KL, Gara M, Marin H, Mark MH, et al. Depression in Parkinson's disease: symptom improvement and residual symptoms after acute pharmacologic management. The American journal of geriatric psychiatry : official journal of the American Association for Geriatric Psychiatry. 2011;19(3):222-9.

6. Dobkin RD, Menza M, Allen LA, Gara MA, Mark MH, Tiu J, et al. Cognitive-behavioral therapy for depression in Parkinson's disease: a randomized, controlled trial. The American journal of psychiatry. 2011;168(10):1066-74.

7. Weintraub D, Mavandadi S, Mamikonyan E, Siderowf AD, Duda JE, Hurtig HI, et al. Atomoxetine for depression and other neuropsychiatric symptoms in Parkinson disease. Neurology. 2010;75(5):448-55. 
8. Barone P, Poewe W, Albrecht S, Debieuvre C, Massey D, Rascol O, et al. Pramipexole for the treatment of depressive symptoms in patients with Parkinson's disease: a randomised, double-blind, placebo-controlled trial. Lancet Neurology. 2010;9(6):573-80.

9. Devos D, Dujardin K, Poirot I, Moreau C, Cottencin O, Thomas P, et al. Comparison of desipramine and citalopram treatments for depression in Parkinson's disease: a double-blind, randomized, placebo-controlled study. Movement Disorders. 2008;23(6):850-7.

10. Menza M, Dobkin RD, Marin H, Mark MH, Gara M, Buyske S, et al. A controlled trial of antidepressants in patients with Parkinson disease and depression. Neurology. 2009;72(10):886-92. 11. Dobkin RD, Menza M, Bienfait KL, Gara M, Marin H, Mark MH, et al. The impact of antidepressant treatment on cognitive functioning in depressed patients with Parkinson's disease. J Neuropsychiatry Clin Neurosci. 2010;22(2):188-95.

12. Paumier KL, Siderowf AD, Auinger P, Oakes D, Madhavan L, Espay AJ, et al. Tricyclic antidepressants delay the need for dopaminergic therapy in early Parkinson's disease. Mov Disord. 2012 (in press).

* This study demonstrates that early PD patients under treatment with tricyclic antidepressants show a significant delay in the initiation of dopaminergic therapy.

13. Richard IH, McDermott MP, Kurlan R, Lyness JM, Como PG, Pearson N, et al. A randomized, double-blind, placebo-controlled trial of antidepressants in Parkinson disease. Neurology. 2012;78(16):1229-36.

** This is the largest randomized controlled study to demonstrate the efficacy and safetyof both paroxetine and venlafaxine in treating depression in Parkinson's disease (Class I evidence).

14. Seppi K, Weintraub D, Coelho M, Perez-Lloret S, Fox SH, Katzenschlager R, et al. The Movement Disorder Society Evidence-Based Medicine Review Update: Treatments for the nonmotor symptoms of Parkinson's disease. Movement disorders : official journal of the Movement Disorder Society. 2011;26 Suppl 3:S42-80. 
15. Pal E, Nagy F, Aschermann Z, Balazs E, Kovacs N. The impact of left prefrontal repetitive transcranial magnetic stimulation on depression in Parkinson's disease: a randomized, double-blind, placebo-controlled study. Movement disorders : official journal of the Movement Disorder Society. 2010;25(14):2311-7.

16. Starkstein S, Merello M, Jorge R, Brockman S, Bruce D, Power B. The syndromal validity and nosological position of apathy in Parkinson's disease. Movement Disorders. 2009;15:1211-6 17. Dujardin K, Sockeel P, Delliaux M, Destee A, Defebvre L. Apathy May Herald Cognitive Decline and Dementia in Parkinson's Disease. Movement Disorders. 2009;24(16):2391-7. 18. Reijnders JSAM, Scholtissen B, Weber WEJ, Aalten P, Verhey FRJ, Leentjens AFG. Neuroanatomical correlates of apathy in Parkinson's disease: A magnetic resonance imaging study using voxel-based morphometry. Movement disorders : official journal of the Movement Disorder Society. 2010;25(14):2318-25.

19. Weintraub D, Hurtig HI. Presentation and management of psychosis in Parkinson's disease and dementia with Lewy bodies. The American journal of psychiatry. 2007;164(10):1491-8.

20. Morgante L, Colosimo C, Antonini A, Marconi R, Meco G, Pederzoli M, et al. Psychosis associated to Parkinson's disease in the early stages: relevance of cognitive decline and depression. Journal of neurology, neurosurgery, and psychiatry. 2012;83(1):76-82.

21. Forsaa EB, Larsen JP, Wentzel-Larsen T, Goetz CG, Stebbins GT, Aarsland D, et al. A 12-year population-based study of psychosis in Parkinson disease. Archives of Neurology. 2010;67(8):9961001.

22. Fernandez HH, Aarsland D, Fenelon G, Friedman JH, Marsh L, Troster Al, et al. Scales to assess psychosis in Parkinson's disease: Critique and recommendations. Movement disorders : official journal of the Movement Disorder Society. 2008;23(4):484-500.

23. Weintraub D, Chen P, Ignacio RV, Mamikonyan E, Kales HC. Patterns and trends in antipsychotic prescribing for Parkinson disease psychosis. Arch Neurol. 2011;68(7):899-904. Epub 2011/07/13. 
** This study reported that about half of patients with Parkinson's disease and psychosis receive antipsychotic medication, with quetiapine being used in two thirds of the patients.

24. Weintraub D, Koester J, Potenza MN, Siderowf AD, Stacy M, Voon V, et al. Impulse control disorders in Parkinson disease: a cross-sectional study of 3090 patients. Archives of Neurology. 2010;67(5):589-95.

25. Avila A, Cardona X, Martin-Baranera M, Bello J, Sastre F. Impulsive and compulsive behaviors in Parkinson's disease: a one-year follow-up study. Journal of the Neurological Sciences. 2011;310(12):197-201.

26. Weintraub D, Sohr M, Potenza MN, Siderowf AD, Stacy M, Voon V, et al. Amantadine use associated with impulse control disorders in Parkinson disease in cross-sectional study. Annals of Neurology. 2010;68(6):963-8.

27. Voon V, Sohr M, Lang AE, Potenza MN, Siderowf AD, Whetteckey J, et al. Impulse control disorders in Parkinson disease: a multicenter case--control study. Annals of Neurology. 2011;69(6):986-96.

** This multicentre case-control studydemonstrates that psychosis in Parkinson's disease is associated with other psychiatric disorders, such as depression, anxiety and obsessive-compulsive symptoms.

28. Cilia R, Cho SS, van Eimeren T, Marotta G, Siri C, Ko JH, et al. Pathological gambling in patients with Parkinson's disease is associated with fronto-striatal disconnection: a path modeling analysis. Movement disorders : official journal of the Movement Disorder Society. 2011;26(2):22533. 